\title{
Encyclopedia
}

\section{Tunable surface wettability of copolymers combined substrate by pulsed plasma}

Octafluorocyclobutane and acrylic acid $\left(\mathrm{C}_{4} \mathrm{~F}_{8}\right.$-co-AA) are plasma copolymerized onto low-density polyethylene (LDPE) and glass slides under various pulsation periods of radio frequency pulsed plasma. The surface wettability of plasma polymer coating is traditionally considered as a substrate independent property. The combined effect of ultrathin $\mathrm{C}_{4} \mathrm{~F}_{8}$-coAA coatings and LDPE substrate on surface wettability presented. The high concentration of the carboxylic acid functional groups gives rise to hydrophilicity via lowering duty cycle and substrate impact give rise to hydrophobicity for ultrathin coatings. The X-ray photoelectron spectroscopy (XPS) and coating thickness measurements confirmed that the sudden increase in water contact angle for the lower duty cycle is influenced by the hydrophobic substrate for ultrathin polymer coatings. It is highlighted that the precise control over the surface wettability is attained by tuning the plasma parameters. The substrate-dependent wettability for flat substrate persisted for longer than 8 weeks, which demonstrates wetting stability for ultrathin coatings.

Further details can be found in the article byl. Muzammil et al. from Korea Institute of Machinery and Materials

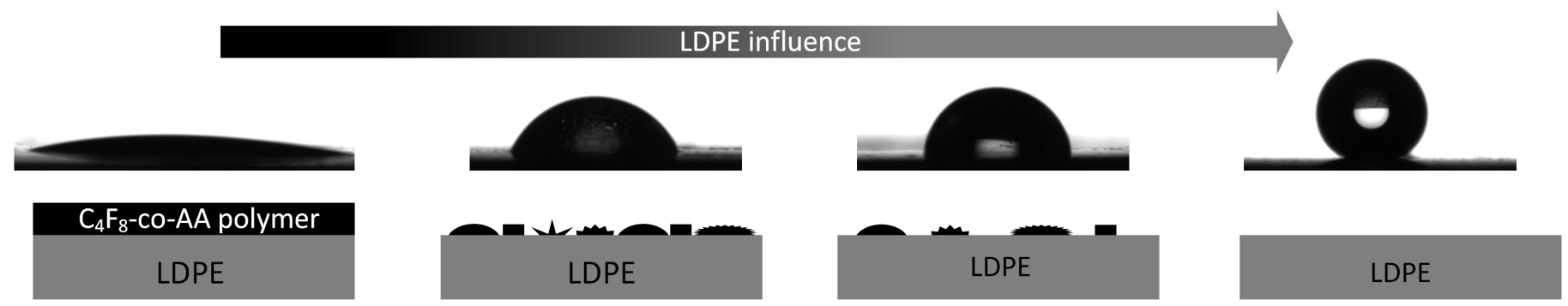

Octafluorocyclobutane and acrylic acid $\left(\mathrm{C}_{4} \mathrm{~F}_{8}\right.$-co-AA) are plasma copolymerized onto low-density polyethylene (LDPE) and glass slides under various pulsation periods of radio frequency pulsed plasma. The surface wettability of plasma polymer coating is traditionally considered as a substrate independent property. The combined effect of ultrathin $\mathrm{C}_{4} \mathrm{~F}_{8}-\mathrm{co}^{-}$ AA coatings and LDPE substrate on surface wettability presented. The high concentration of the carboxylic acid functional groups gives rise to hydrophilicity via lowering duty cycle and substrate impact give rise to hydrophobicity for ultrathin coatings. The X-ray photoelectron spectroscopy (XPS) and coating thickness measurements confirmed that the sudden increase in water contact angle for the lower duty cycle is influenced by the hydrophobic substrate for ultrathin polymer coatings. It is highlighted that the precise control over the surface wettability is attained by tuning the plasma parameters. The substrate-dependent wettability for flat substrate persisted for longer than 8 weeks, which demonstrates wetting stability for ultrathin coatings. [1]

Further details can be found in the article byl. Muzammil et al. from Korea Institute of Machinery and Materials

\section{References}

1. I. Muzammil; Y. P. Li; X. Y. Li; D. K. Dinh; M. Imran; H. Sattar; M. K. Lei; Tunable surface chemistry and wettability of octafluorocyclobutane and acrylic acid copolymer combined LDPE substrate by pulsed plasma polymerization. Journal of Coatings Technology and Research 2019, 20, 20, 10.1007/s11998-019-00244-z.

\section{Keywords}

Tunable Surface Chemistry; Tunable Surface Wettability; Octafluorocyclobutane; Acrylic Acid; LDPE; Pulsed Plasma; Plasma Polymerization; Muzammil Plasma; Iqbal Plasma; Plasma Engineering; Surface Engineering; Korea; China; Materials Modification; Surface Science; Superh 
c) (i) (C) 2019 by the author(s). Distribute under aCreative Commans CC BY license 